\title{
Vulnerabilidad social en tiempos del COVID 19, leyes e impacto sobre la pobreza
}

\section{Social vulnerability in times of COVID 19, laws and impact on poverty}

\author{
(iD) \\ Alfredo R. Anaya Narváez \\ Universidad de Córdoba, Colombia \\ aranaya@correo.unicordoba.edu.co

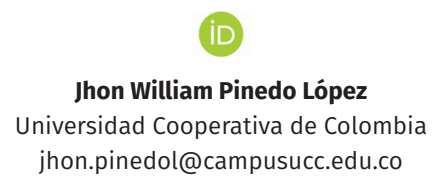 \\ William Alejandro Niebles Nuñez \\ Universidad de Sucre, Colombia \\ william.niebles@unisucre.edu.co
}

Recibido: 01 de julio de 2021 / Aceptado: 11 de noviembre de 2021 https://doi.org/10.17081/just.26.40.5155

\begin{abstract}
Resumen
Objetivo: Establecer la vulnerabilidad de cada estrato socioeconómico del municipio de Sincelejo y el papel del marco jurídico colombiano en términos de probabilidades de caer en situación de pobreza monetaria por causa de deterioro de los ingresos laborales provocado por eventos económicos adversos, inducidos por otros fenómenos tal como la actual expansión del Covid 19. Método: Estimación de dos modelos econométricos Logit con información proveniente de 541 encuestas del tipo calidad de vida, aplicadas al mismo número de hogares del municipio de Sincelejo en el año 2017. Resultados: Los modelos estimados resultan adecuados por cuanto los estimadores, considerados individualmente, resultan estadísticamente significativos con un nivel de confianza mayor del $99 \%$, así como también resulta estadísticamente significativo, con el mismo nivel de confianza, el modelo globalmente considerado, ya que el Estadístico LR tiene un valor $p<0,01$. En adición, el ajuste de los dos modelos en bueno debido a que los porcentajes de predicciones correctas son $85,03 \%$ y 79,45\%. Conclusiones: La evidencia empírica demuestra, aunque todas las personas están expuestas a fenómenos económicos desfavorables, los del estrato bajo tienen mayores probabilidades de quedar en condiciones de pobreza, es decir tienen alto grado de vulnerabilidad y se confirma una relación inversa entre los estratos socioeconómicos y los grados de vulnerabilidad. Por otra parte, las leyes colombianas han sido ineficaces para resolver los problemas asociados a la pobreza y por tanto, si se desea garantizar una reducción sostenida de la misma se hace necesario que las políticas públicas se hagan efectivas y se enfoquen tanto en la población en condiciones de pobreza como en la vulnerable.
\end{abstract}

Palabras CLAVes: Vulnerabilidad, pobreza monetaria, Covid 19, leyes, Logit. 


\begin{abstract}
Objective: To establish the vulnerability of each socioeconomic stratum of the municipality of Sincelejo in terms of probabilities of falling into monetary poverty due to deterioration of labor income caused by adverse economic events, induced by other phenomena such as the current expansion of Covid 19. Method: Estimation of two econometric Logit models with information from 541 surveys of the quality of life type, applied to the same number of households in the municipality of Sincelejo in 2017. Results: The estimated models are adequate because the estimators, considered individually, are statistically significant with a confidence level greater than $99 \%$, as well as the model considered globally is statistically significant with the same confidence level, since the LR statistic has a $p<0.01$ value. In addition, the fit of the two models in good because the percentages of correct predictions are $85.03 \%$ and $79.45 \%$. Conclusions: The empirical evidence shows, although all people are exposed to unfavorable economic phenomena, those in the low stratum are more likely to be left in conditions of poverty, i.e. they have a high degree of vulnerability and an inverse relationship between socioeconomic strata and degrees of vulnerability is confirmed. On the other hand, Colombian laws have been ineffective in solving the problems associated with poverty and therefore, if we wish to guarantee a sustained reduction of poverty, it is necessary that public policies become effective and focus on both the poor and the vulnerable population.
\end{abstract}

KEYWORDS: Vulnerability, monetary poverty, Covid 19, laws, Logit.

Como Citar: Anaya Narváez, A. R., Pinedo López, J. W., \& Niebles Nuñez, W. A. (2021). Vulnerabilidad social en tiempos del COVID 19, leyes e impacto sobre la pobreza. Justicia, 26(40), 168-187. https://doi.org/10.17081/ iust.26.40.5155

\title{
I. Introducción
}

Este articulo tiene por objeto determinar las probabilidades que tienen las personas de los diferentes estratos socioeconómicos del municipio de Sincelejo, que sin estar en condición de pobreza monetaria pueden caer a dicha condición ante la pérdida de empleo con la consiguiente disminución de sus ingresos laborales; pérdida de empleo que pude darse en el perceptor principal del hogar y/o de otros perceptores, particularmente como consecuencia de crisis económicas provenientes de los ciclos económicos o de fenómenos inesperados como la pandemia ocasionada por la COVID 19.

Acogiendo la propuesta de (CEPAL, 2019), los estratos socioeconómicos en que se divide la población según sus niveles de ingresos, expresados en números de líneas de pobreza, son los que se indican en la tabla 1, lo que implica que entre más bajo se ubique el estrato, menos ingresos per cápita registra.

TABLA 1. ESTRATOS SOCIOECONÓMICOS SEGÚN INGRESOS

\begin{tabular}{c|cc}
\hline \multicolumn{2}{|c}{ Estrato } & Ingreso \\
\hline \multirow{3}{*}{ Bajos } & Pobreza Extrema & $<$ Línea de pobreza extrema [LPE] \\
& Pobreza & $>1$ LPE $y<$ Línea de pobreza [LP] \\
& Bajos no pobres & $>1 \mathrm{y}<1,8 \mathrm{LP}$ \\
Medios & Medios bajos & 1,8 a 3 LP \\
& Medios intermedios & $>3$ a 6 LP \\
Alto & Medios altos & $>6$ a 10 LP \\
\hline
\end{tabular}

Fuente: Elaboración propia con base en CEPAL (2019) 
Por otra parte, la vulnerabilidad que aquí se tratará se enmarca en el concepto de vulnerabilidad social relacionada con la pobreza, específicamente con la pobreza monetaria; por lo cual es importante anotar que, de acuerdo con (Wood et al., 2010) este concepto ha venido siendo tratado de manera relativamente reciente, dado que su uso inicial se refería a las fragilidades de la población expuesta a fenómenos naturales potencialmente destructivos.

Ahora bien, (Nuñez \& Espinosa, 2005) y (CEPAL, 2019) indican que en principio todas las personas que no se encuentran en condición de pobreza monetaria, son vulnerables a caer en esa situación ante eventos adversos de orden económico; sin embargo, eventualmente las probabilidades son diferentes para distintos grupos poblacionales, los cuales dependen de las capacidades de cada uno de ellos de afrontarlos, entre las que se encuentra por ejemplo, la capacidad financiera relacionada con los ingresos; por lo cual resulta lícito hablar de grados de vulnerabilidad. Por tanto, este trabajo pretende establecer la vulnerabilidad de cada estrato en términos de probabilidades de caer en situación de pobreza monetaria por causa de deterioro de los ingresos laborales provocado por eventos económicos adversos.

Como punto de partida y para tener una buena aproximación a la situación socioeconómica en el municipio de Sincelejo, reflejada en la composición estratificada de su población, se presenta en la figura 1 un comparativo de (4) entidades (2 países, América Latina y el municipio de Sincelejo) en el año 2017, en la que se registran detalles como que la pobreza extrema prácticamente no existe en Uruguay $[0,1 \%]$ y donde su pobreza se encuentra en un bajísimo nivel del $2,6 \%$, mientras que sus estratos medios y alto concentran el $82,7 \%$ de su población.

Figura 1.Población según estratos por ingresos per cápita 2017

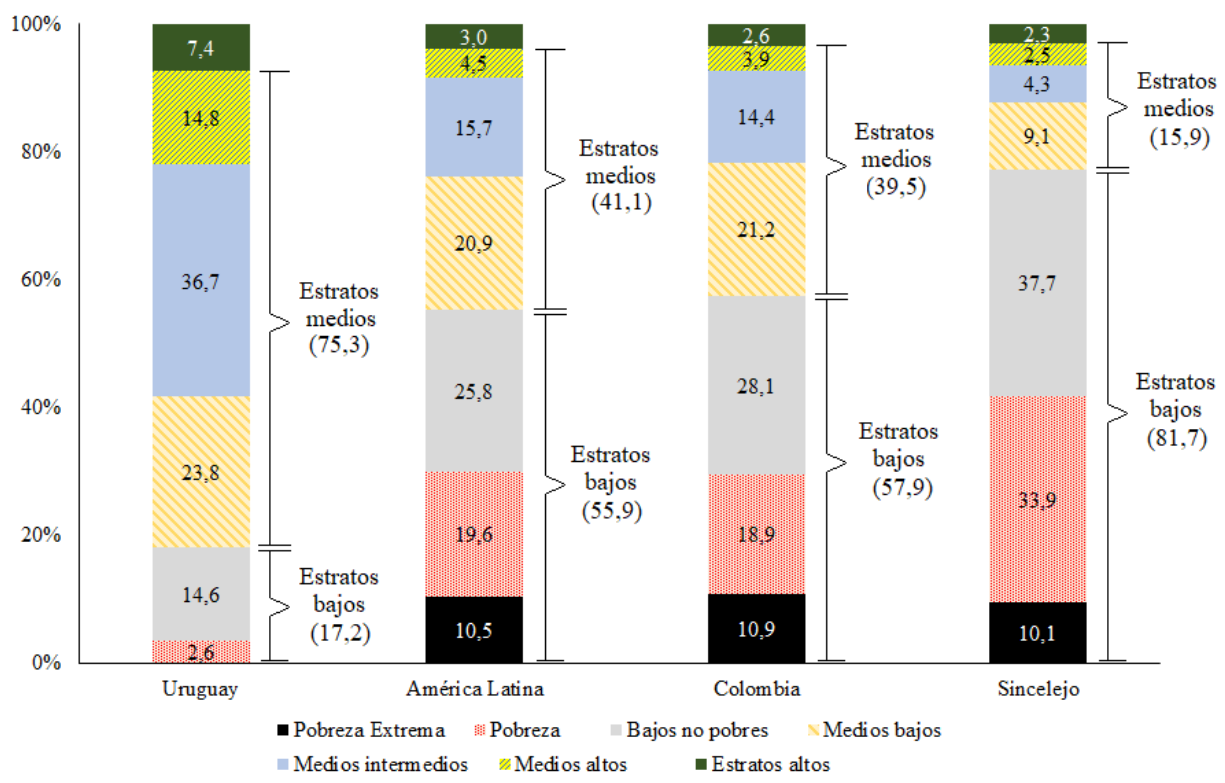

Fuente: Elaboración propia con base en (CEPAL, 2019)

En contraste, Colombia ostenta cifras de pobreza superiores al promedio latinoamericano y, en su conjunto, la pobreza y la pobreza extrema constituyen casi el $30 \%$ de su población mientras que las clases medias y alta suman el 42,1\%. La situación del municipio de Sincelejo en esta materia, que se puede calificar de severa en materia de pobreza, es la opuesta a la de Uruguay por cuanto los estratos bajos acaparan el $81,7 \%$ de su población y el estrato bajo no pobre participa con el $37,7 \%$. 
Adicionalmente, considerando que los ingresos familiares, que son los que determinan la pobreza monetaria, provienen de fuentes diversas, es importante conocer la participación de los ingresos laborales con el fin de estimar la afectación de un evento adverso de tipo económico sobre la pobreza monetaria vía reducción de tales ingresos. La figura 2 muestra un comparativo de esta participación entre los hogares de Sincelejo con América Latina [promedio de 18 países], en donde se aprecia que en todos los estratos municipales se registra un mayor guarismo, lo que sugiere una mayor dependencia de estos recursos en los hogares sincelejanos. Así, por ejemplo, mientras que en América Latina el promedio general es del orden del $62 \%$ y de los estratos bajos no pobres promedian el $68 \%$, en el municipio de Sincelejo son del $82 \%$ y $81 \%$ respectivamente.

Figura 2. Ingreso por trabajo según estrato (\% del ingreso total)

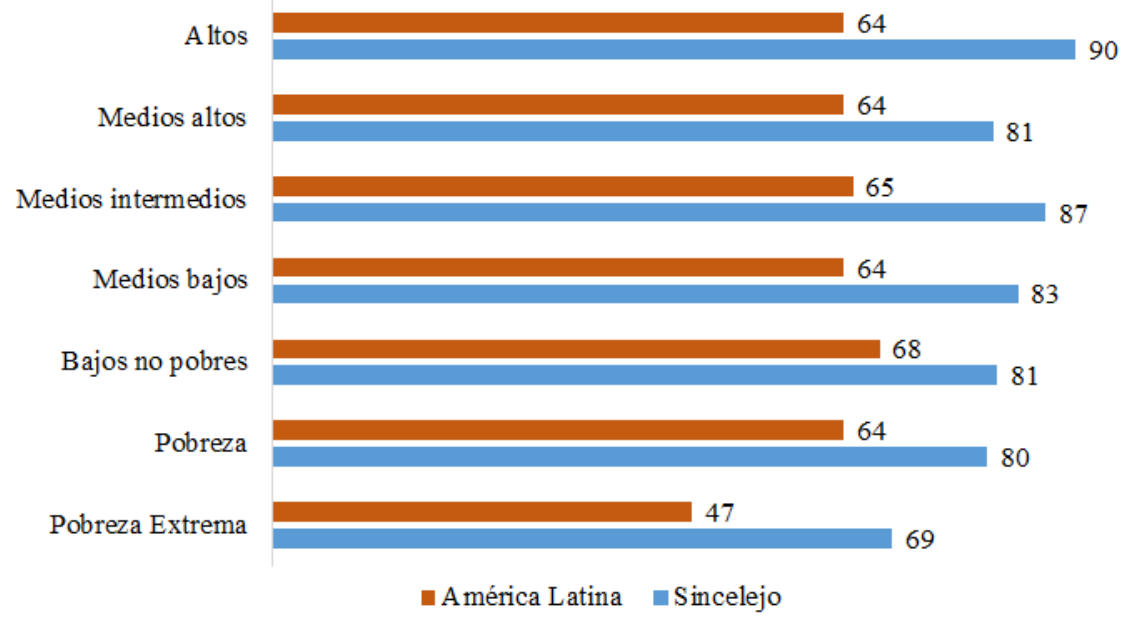

Fuente: Elaboración propia con base en encuesta propia (2017) y (CEPAL, 2019)

Con todo lo anterior, considerando los altos índices de pobreza en Sincelejo y teniendo en cuenta que la tasa de población ubicada en el estrato bajo no pobre, la cual se encuentra más próximo al estrato en condición de pobreza, es muy alta; así como también la alta dependencia que tienen los ingresos familiares de los ingresos laborales; resulta clara la importancia de establecer las probabilidades de quedar en condición de pobreza de los diferentes estratos que no se encuentran en ella y de esa manera determinar las vulnerabilidades de cada uno de los mencionados estratos ante disminuciones de los ingresos laborales.

Para América latina estas probabilidades se muestran de manera discriminada en la figura 3, la cual revela que para todos los estratos, las mayores probabilidades de caer en situación de pobreza la tienen personas que pertenecen a hogares en que el perceptor principal del hogar se queda sin ingresos y las menores aquellos en que los perceptores secundarios se queden sin ingresos, en razón a que el ingreso laboral promedio del perceptor principal sistemáticamente supera de forma significativa el ingreso laboral promedio de los perceptores adicionales. Por supuesto, también se observa que, para los dos casos, las probabilidades son mayores para las personas que se encuentran clasificados en estratos bajos, seguidos por los de estratos medios y por último los de estratos altos (CEPAL, 2019).

Pues bien, para adelantar la tarea propuesta se aplicará un modelo econométrico probabilístico Logit utilizando la información recabada en el municipio de Sincelejo en junio del año 2017 mediante la aplicación de 541 encuestas tipo calidad de vida. 
Figura 3. Riesgo de caer en situación de pobreza en América Latina

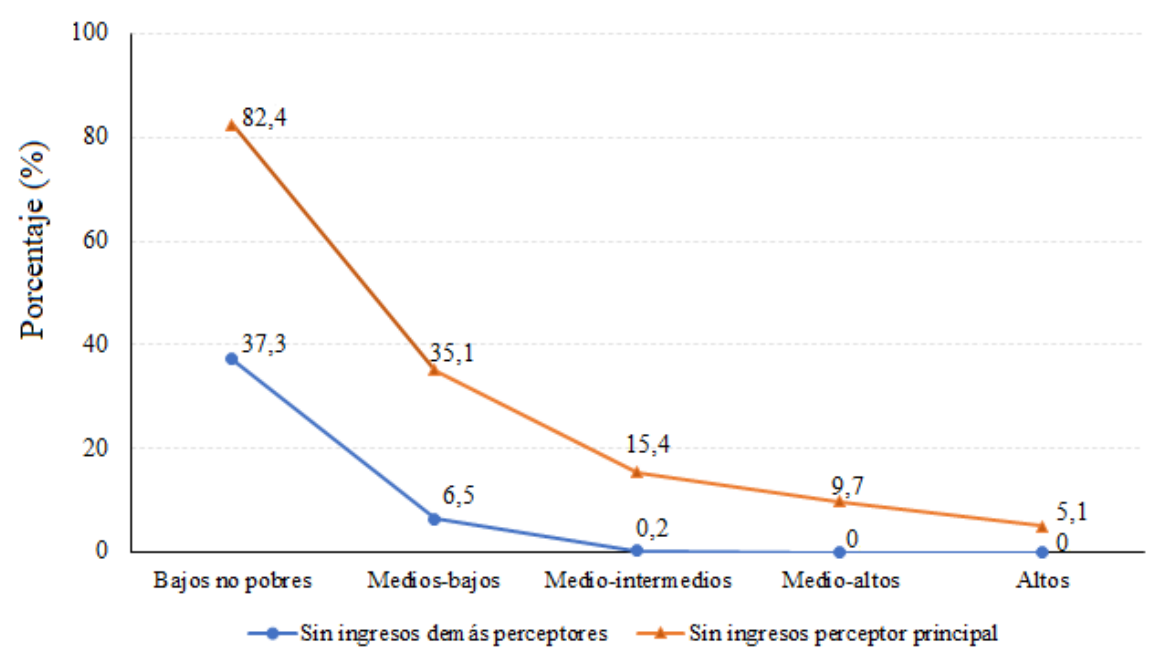

Fuente: Elaboración propia con base en (CEPAL, 2019)

Los resultados de esta investigación demuestran que, aun cuando todas las personas están expuestos a choques adversos, los estratos bajos no pobres son altamente vulnerables por cuanto tienen mayores probabilidades de quedar en condiciones de pobreza ante choques que afecten la actividad económica, en tanto que los demás tienen vulnerabilidades medias y bajas respectivamente, denotándose una relación inversa entre ellas. Para adelantar la tarea propuesta se procederá seguidamente a exponer sucintamente la conceptualización y aportes de distintos autores sobre la vulnerabilidad, la pobreza y los ingresos, así como la relación entre ellas.

\subsection{La vulnerabilidad}

La vulnerabilidad es un concepto que en la actualidad acoge múltiples dimensiones, pero que ha sido abordado tradicionalmente desde el campo de las ingenierías y está asociado con la afectación de ciertos grupos poblacionales por fenómenos naturales, por lo cual desde las ciencias naturales y las ciencias aplicadas se contribuye con el diseño de modelos matemáticos que se utilizan para aminorar el impacto de tales amenazas (Wood et al., 2010). Sin embargo, desde hace poco más de veinte años este tema se ha venido tratando en el ámbito socioeconómico dando paso al concepto de vulnerabilidad social con trabajos como el de Otto, (Castelao, Massot \& Eriz, 2014) que estudia la desprotección en que se encuentran algunos grupos poblacionales frente a fenómenos externos que les pueden causar daños; así como los de (Moser, 1999) y (Mora \& Pérez, 2006), que han examinado la vulnerabilidad de personas y grupos desde sus condiciones de pobreza, desigualdad social y exclusión.

El concepto de vulnerabilidad en su acepción social por tanto está asociado a la fragilidad y a la debilidad de ciertos grupos poblacionales, causada por la insuficiencia de instrumentos o herramientas, de orden financiero, físico, social y humano, que no les permiten afrontar exitosamente eventos adversos de tipo económico y por tanto sufren sus consecuencias en detrimento de su situación previa (Busso, 2005). Cuando los sucesos desfavorables son crisis económicas, la vulnerabilidad se encuentra relacionada con los riesgos que tienen los individuos que no están en condiciones de pobreza de caer en ese estado, sin desconocer que, según (Nuñez \& Espinosa, 2005), aunque todas las personas pueden ser vulnerables, "los individuos pobres son mucho más" (p.3); lo cual revela la íntima relación entre vulnerabilidad y pobreza. 
En este punto resulta importante indicar que (Naciones Unidas, 2004) a través de la oficina de asuntos económicos y sociales, propuso la siguiente definición:

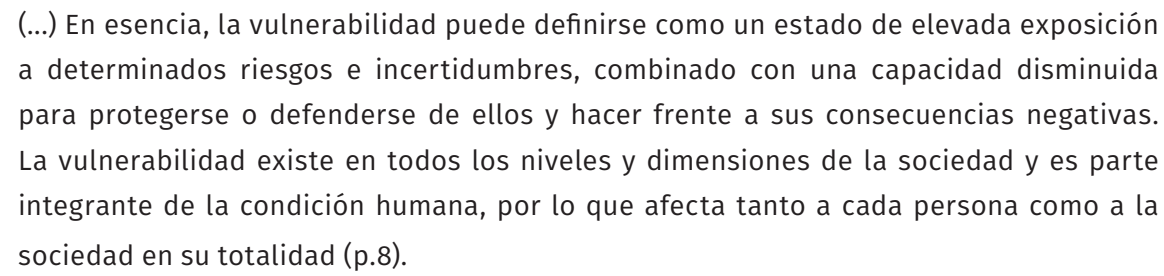

Según (Durán, 2017) existen diferentes definiciones de vulnerabilidad, provenientes de las diferentes concepciones epistemológicas en la que se enmarcan, así como de las metodologías utilizadas, por lo cual se hace necesario precisar que este artículo lo aborda desde la perspectiva socioeconómica y por tanto la vulnerabilidad se refiere a la exposición a eventos desfavorables de tipo económico de los hogares y personas, así como a las posibilidades que tienen para afrontarlos exitosamente. Concretamente, en este trabajo y siguiendo a (Miranda, 2020), la vulnerabilidad vendrá dada por la las probabilidades que tienen diferentes grupos poblacionales, que no son pobres, de caer en la pobreza por causa de eventos o choques externos adversos que afecten la actividad económica. Se trata de la debilidad de los hogares que no se encuentran en condición de pobreza monetaria, especialmente de estratos bajos según la definición de (CEPAL, 2019), para afrontar crisis económicas,

Ahora bien, en principio, según (Nuñez \& Espinosa, 2005), todas las personas que no se encuentran en condición de pobreza monetaria, independientemente del estrato socioeconómico al que pertenezcan, tienen riesgos de quedar en esa condición en presencia de eventos económicos adversos, es decir son vulnerables. Sin embargo, eventualmente las probabilidades de cada estrato son distintas y tienden a disminuir en la medida en que los estratos sean más altos por cuanto es mayor su capacidad de respuesta ante tales fenómenos derivada de los instrumentos de que dispone (Nuñez \& Espinosa, 2005; CEPAL, 2020).

En ese orden, la vulnerabilidad es una cuestión de grados y por tanto en este trabajo se adoptarán términos como alto, medio o bajo grado de vulnerabilidad. De esa manera, a priori se espera que la población de los estratos bajos no pobres de sincelejo, acusen las mayores probabilidades de caer en pobreza y por tanto se clasifiquen como los más vulnerables corroborándose de paso una relación inversa entre los estratos socioeconómicos y los niveles de vulnerabilidad.

\subsection{La pobreza}

La pobreza es un fenómeno que viene afectando e inquietando a la sociedad desde hace muchos siglos, y muestra de ello, es la mención reiterada que se hace de esta en libros tan antiguos como la Biblia y el Corán (Casas \& Barichello, 2015). Es objeto de estudio para investigadores de diferentes disciplinas científicas, entre las que se encuentran la economía, sociología, antropología, derecho y en las últimas décadas, la psicología social, que se dedica a indagar sobre percepción de la pobreza (Marduk, González \& Malavé, 2010).

Generalmente, la pobreza es asociada a carencia de bienes básicos, insuficiencia en el ingreso y a la falta de oportunidades. (Sen, 2000) la define como el estado de privación del bienestar, concretamente de alimentación, vivienda, educación y salud, mientras que el (Banco Mundial, 1990) la relaciona con la incapacidad que tienen las personas para lograr un nivel de vida mínimo. En cambio, para (Martínez, 2015) es la privación material, medida a través del ingreso o del consumo individual o familiar. 
Sobre el origen de la pobreza de muchos países, hay varias teorías e hipótesis formuladas: el saqueo de países colonizadores; la ignorancia; la falta de recursos naturales; la ubicación geográfica y la cultura, entre otras (Acemoglu \& Robinson, 2012). Para (Sachs, 2008) la pobreza tiene varios niveles: pobreza extrema, pobreza moderada y pobreza relativa.

Ahora bien, la pobreza es analizada desde paradigmas y percepciones diferentes, que buscan identificar sus causas, conceptualizarla o medirla, asociándola a carencia de capacidades y oportunidades o a causas estructurales, individuales o de género (Mayorga, 2018). En este sentido, (Casas \& Barichello, 2015) sostienen que se han desarrollado tres concepciones de la pobreza: subsistencia, necesidades básicas y privación relativa.

Aunque es examinada desde diferentes disciplinas y percepciones, se considera que la investigación sobre la pobreza es dominada actualmente por economistas neoclásicos, quienes la miden cuantitativamente a partir del ingreso y parten del supuesto de que el bienestar es el elemento constitutivo del nivel de vida (Jaimez, 2015). Sin embargo, es pertinente anotar que el trabajo de medición de la pobreza que realizan economistas neoclásicos se encuentra acompañado de importantes estudios que aportan notablemente a la comprensión de este fenómeno o formulan interrogantes que invitan a la reflexión y al debate académico, entre los cuales sobresale el de (Miller, 2001), que en su calidad de cooperante para el desarrollo en naciones afectadas por la pobreza, establece una tipología de pobres, con el fin de dignificarlos: 1) pobres dignos de ayuda, en los que incluye a las viudas, huérfanos, discapacitados y ancianos abandonados, quienes a su juicio trabajarían si pudieran; 2) los trabajadores pobres; conformados por personas que tienen la capacidad y están dispuestos a trabajar, pero que requieren ayuda, asociada a la inversión, asesoría y acompañamiento, y 3) los pobres que no merecen ayuda, grupo conformado por personas que tienen la capacidad, pero no quieren trabajar. Como responsable de asignación de recursos financieros y a partir de su experiencia como cooperante para el desarrollo, (Miller, 2001) sostiene lo siguiente de aquellos que se niegan a trabajar:

\footnotetext{
Ellos no merecen nuestros donativos, sino nuestra amorosa confrontación. Dar indiscriminadamente a los pobres que no lo merecen es aumentar su pobreza. Fracasar en desafiarlos es condenarlos a su pobreza. Debemos hacer todo lo posible para restaurar y mantener la dignidad de los pobres, independientemente de la categoría en la que caigan (p. 246).
}

Este autor también reconoce que el trabajo es una de las herramientas claves para sacar a la gente de la pobreza. Sin embargo, evidencia que muchos pobres son trabajadores esforzados, que trabajan en jornadas de hasta 16 horas al día, pero a consideración de él puede suceder que otros roban el fruto de su labor, amparados en comportamientos corruptos o que la contribución que su trabajo aporta a la comunidad sea subvalorada (Miller, 2001). Así mismo, formula un interrogante para la reflexión “¿Estamos tratando a los pobres como portadores de la imagen divina, con imaginación para descubrir y crear recursos o cómo a una clase de personas que representan simplemente bocas que llenar?" (p.8).

En una línea similar, (Mangalwadi, 2009), asocia la pobreza con el relativismo moral y la corrupción, enfatizando en dos aspectos que hunden a las naciones en la pobreza: no codiciar los bienes del prójimo y no hurtar. Sostiene que los economistas planifican el desarrollo económico, pero no entienden ni tienen en cuenta estos dos aspectos, que obstaculizan dicho desarrollo. "Los economistas saben que la corrupción causa pobreza, pero carecen de armazón intelectual y de recursos espirituales para ayudar a las naciones corruptas a plantearse difíciles cuestiones culturales" (p.35). 
En una perspectiva análoga, (Colson \& Pearcey, 2000) sostienen que la pobreza es un problema de patrones y conductas que no se puede resolver únicamente con programas gubernamentales bien pensados y bien financiados. Para ellos es fundamental que se trate a los seres humanos como agentes morales, a quienes se les debe tatar con el lenguaje del deber y las responsabilidades.

Cuando las cosas van mal, cuando la pobreza y la delincuencia demuestran ser intratables, la presuposición es que el estado no está haciendo lo suficiente. Es así que se ha engendrado una mentalidad de sentirse con derechos, donde los ciudadanos creen que el gobierno les debe apoyo, aunque ellos no cumplan los deberes básicos de la ciudadanía - o aún si participan en comportamiento nocivo o ilegal. ¿Consumen drogas? ¿Son alcohólicos? ¿Son aptos para el trabajo, pero se niegan a trabajar? ¿Están teniendo hijos sin la más mínima intención de mantenerlos económicamente? No importa, igualmente tienen derecho a los beneficios del gobierno y no hay que hacer preguntas. De modo que estos patrones de conducta disfuncionales se robustecen y el ciclo continúa. A los ciudadanos no se los alienta a asumir responsabilidad moral o personal por su vida y decisiones (p.168).

Igualmente, manifiestan que, al no alentar a los ciudadanos a asumir responsabilidad moral, el estado providencia ha fracasado, creando una clase social dependiente y también una cantidad de patologías sociales que la acompañan, desde familias destruidas y adolescentes embarazadas hasta abuso de drogas y delincuencia, que reproducen desproporcionadamente la pobreza.

Otra posición interesante es la de (Cope, 2010), quien considera que la redistribución es una aparente solución, por cuanto se supone que el problema de la pobreza se resolvería si los que tienen dan algo a los que no tienen. No obstante, argumenta que en la práctica esto resulta contra productivo, porque engendra más pobreza. En este caso, concluye que debe primar la oportunidad antes que la ayuda, y que esta última debe reservarse solo para los que no tienen forma de proveer para sí mismos. En ese mismo sentido (Klisberg, 2011) se opone a que las soluciones a esta problemática se enfoquen en ayudas a manera de subsidios, y considera que el estado debe adelantar y ejecutar programas encaminados a dar solución estructural al problema de la pobreza de manera efectiva.

En esta misma dirección apunta la propuesta de (Sen, 2000), que considera que la solución debe pasar por la adopción de políticas sociales que promuevan la dotación y expansión de las capacidades básicas de las personas, de tal manera que se les posibilite ser autosuficientes y vivir una vida plena en el marco de la libertad de poder elegir entre diferentes alternativas, es decir de decidir cómo organizar su vida. Acogiendo esta concepción El PNUD formuló el IPH Índice de Pobreza Humana.

Además de lo anterior, existen posturas como la de (Pérez, 2010), enmarcadas en una concepción progresista de la pobreza, que insisten en la obligación que tienen los estados de garantizar los derechos fundamentales de las personas, de tal manera que se les posibilite llevar una vida adecuada y por esa vía reducir la pobreza. En ese sentido indica que,

El analfabetismo, la malnutrición, el hambre, la muerte por inanición o por falta de asistencia médica para atender enfermedades prevenibles y curables, no es una expresión de la mala suerte o de una actitud poco proactiva del individuo para llevar una vida digna: son una expresión de ausencia de medidas del Estado para garantizar a todas las personas, sin discriminación alguna, derechos fundamentales" (p.7). 
Por su parte, (Beliles, 2017), sostiene que las razones principales por las que las naciones están en la pobreza son la falta de recursos espirituales y la ignorancia. Considera que la provisión de los pobres no es responsabilidad del Estado, sino de individuos, familias, comunidades y la Iglesia. No cumplir esta responsabilidad, trae consecuencias en el imaginario de la sociedad y en el papel del Estado:

Cuando el Estado asume esta responsabilidad, la Iglesia y los individuos eventualmente creerán que el gobierno debe cuidar a los pobres. Muchas naciones en las últimas décadas demuestran este punto. Mientras que el dinero del gobierno gastado en el bienestar ha aumentado dramáticamente, también ha aumentado la pobreza de la nación. La gran mayoría de cada dólar asignado a los pobres y necesitados es consumido por la burocracia gubernamental que no tiene intención de solucionar un problema. El dinero del gobierno no es la solución para la pobreza (p.27).

Para este autor, una economía cristiana es una solución real a la pobreza, porque bajo el cumplimiento de sus principios sucede lo siguiente: los hombres respetan la propiedad del otro; no se engañan mutuamente; cumplen con los contratos; honestidad en el ejercicio del poder político o económico; cada persona utiliza los talentos y los pone al servicio de la sociedad; derechos individuales, tales como ingresar en una ocupación de elección personal, iniciar un negocio y comprar los bienes que uno prefiere; responsabilidades económicas individuales de proveer para sí mismo, la familia y los pobres, en lugar de depender del gobierno civil para satisfacer las necesidades; poder para crear riquezas - trabajar, hacer negocios, comercializar con integridad y servicio para ganar riquezas, prosperidad e influencia cultural y compasión genuina; es decir, familias, negocios e iglesias dando y cuidando a los ancianos, pobres, y victimas de adversidades. Esto significa, una economía de principios y valores, que antepone el trabajo, la solidaridad y honestidad a la codicia, pereza y egoísmo, que actualmente prevalece.

Como se puede evidenciar, los autores (Miller, 2011; Mangalwadi, 2009; Colson \& Pearcey, 2000; Cope, 2010; Beliles, 2017), realizan una mirada cualitativa muy pertinente, que no riñe con los hallazgos cuantitativos obtenidos a partir de las mediciones realizadas de manera monetaria o multidimensional que se hacen a nivel internacional y que en Colombia son oficialmente las dos formas utilizadas para medir la pobreza (Anaya, Buelvas \& Valencia, 2015). Igualmente, coinciden con quienes incluyen en esta categoría no solo a los excluidos tradicionalmente del mercado del trabajo, sino a los que enfrentan situaciones de precariedad laboral y bajos salarios, informalidad, subempleo y desempleo (Barba, 2009).

\subsection{La justicia social y el derecho}

La pobreza es un fenómeno indeseable que se estudia desde el punto de vista económico en el marco de la economía del bienestar, fundamentado en los análisis de la eficiencia y la equidad y concretamente basado en el segundo teorema de la economía del bienestar que plantea el dilema de la distribución y por tanto motiva la discusión sobre la eficiencia y la equidad que surgen en un problema económico. (Nicholson y Snyder, 2015).

Así las cosas, la manera de cómo viven los miembros de una sociedad depende de cómo se zanje el problema entre eficiencia y equidad, y ello depende de las opciones que tomen tanto los individuos como de las acciones que ejecute el gobierno. "Con las intervenciones gubernamentales a través de sus diferentes programas, que permiten distribuir de nuevo las dotaciones iniciales, o negándose a hacerlo, los gobiernos implícitamente están haciendo juicios de valor sobre cuales miembros de la sociedad deben tener relativamente más bienes" (Perloff, 2012, p. 320). 
Por tanto, La pobreza surge en el marco de la inequidad y por tanto se convierte en un problema de justicia social que debería ser resuelto por el estado, garantizado derechos mediante la aplicación efectiva de normas que los regulen. Lo anterior es consistente con la postura de (Colson y Pearcey, 2000), quienes aseguran que una economía no es un mecanismo autónomo, por cuanto depende de un marco jurídico que mantenga una moneda segura, proteja la propiedad privada, haga cumplir contratos, restrinja la corrupción y contribuya a la prosperidad económica. En este sentido, para que una sociedad prospere económicamente, necesita tener un sistema legal justo, asociaciones políticas sujetas a relaciones colegiadas, y políticas gubernamentales estables y racionales, que deben ser garantizadas por el Estado (Miller,2001).

En Colombia, unos de los fines esenciales del Estado que consagra la Constitución Política son, entre otros, "promover la prosperidad general" y "facilitar la participación de todos en la vida económica" (Art. 2). Estos fines son diseñados bajo la perspectiva de la justicia económica y para ello se establecen algunos deberes del Estado, que tienen como propósitos mejorar el ingreso, calidad de vida y el acceso de todas las personas, especialmente las de menores ingresos, a los bienes y servicios básicos (Arts. 64 y 334 ).

Infortunadamente, el marco constitucional y normativo no ha sido suficiente para lograr la justicia económica y evitar de esta manera los elevados indicadores de pobreza monetaria en el país asociados con altos niveles de informalidad laboral en la economía. Las leyes aprobadas no logran hasta el momento un aprovechamiento óptimo del recurso humano, mediante el empleo y aumento de la productividad, así como tampoco una reducción significativa de la corrupción que empobrece a la nación, ni de la informalidad laboral. Al respecto, (Miller, 2001) sostiene que la estructura de una sociedad viene en última instancia, no de las leyes escritas o de las constituciones, sino de las decisiones morales de sus miembros.

Tanto la pobreza como el desempleo y la informalidad laboral se intentan combatir a través de diferentes leyes. Una de ellas, el documento Conpes 091 de 2005 en el cual se señala como primer objetivo de los compromisos del gobierno colombiano el de erradicar la pobreza extrema y el hambre; y otra es la Ley 1429 de 2010 de formalización y generación de empleo, en la que se pretende además disminuir los altos niveles de informalidad laboral en el país. Dicha ley, define la informalidad por subsistencia como "aquella que se caracteriza por el ejercicio de una actividad por fuera de los parámetros legalmente constituidos, por un individuo, familia o núcleo social para poder garantizar su mínimo vital". Sin embargo, los altos costos de la formalización siguen siendo un obstáculo para lograr el aumento de empleo y la disminución de la pobreza monetaria.

\subsection{Instrumentos jurídicos orientadas al desempleo.}

El estado colombiano, en aras de atenuar la pobreza por causa de desempleo, ha generado varias leyes o decretos que favorecen el resarcimiento del estado de indefensión y vulnerabilidad en que quedan las familias luego de quedar cesantes de una relación laboral o por no poder acceder al mercado laboral. En ese sentido, la constitución de 1991 en su artículo 25 declara que el trabajo es un derecho de todo ciudadano y debe ser protegido, por lo que se infiere, que su propósito es que las familias no queden en estado de vulnerabilidad y por tanto, se generó un marco normativo orientado al cumplimiento de este propósito.

Así, la (Ley 789 de 2002) ofreció lineamientos para la generación de empleo a través de la asignación de rubro de las contribuciones parafiscales administrada por las cajas de compensaciones y modificó el código sustantivo del trabajo para ampliar la protección del estado a los derechos de protección social en una relación laboral. Por su parte, la Ley 1429 de 2010, se orientó a formalizar los empleos provenientes de empresas Informales, a ofrecer lineamientos que conduzcan a crear más empleo formal y a su vez a mejorar los ingresos de estos sectores del mercado laboral (Merchán 2011). 
Del mismo modo, la ley 1636 de 2013 fue creada con la intención de ofrecer un mecanismo de ingreso para el cesante por motivo de perdida de trabajo formal y brindarle una herramienta a través de la oficina de empleo para la reinserción en el mercado laboral. Por último, la (Ley 1780 de 2016), que busca fomentar la creación empresa en una población menor a 35 años de edad y la generación de empleo en este segmento del mercado laboral.

\subsection{Las medidas de la pobreza}

En relación con las medidas de la pobreza, se puede indicar que existen tantas como concepciones y enfoques sobre este fenómeno. En el campo económico, las medidas de la pobreza pueden clasificarse inicialmente en objetivas y subjetivas; así mismo, las objetivas pueden dividirse entre directas e indirectas, y dentro de las directas se encuentran el índice de Necesidades Básicas Insatisfechas [NBI] en tanto que dentro de las indirectas se cuenta con el índice de Pobreza Monetaria [PM] (Busso, 2005)'.

Según (DANE, 2020), en Colombia existen dos medidas oficiales de pobreza ambas objetivas, la primera de ellas es directa y se denomina el Índice de Pobreza Multidimensional [IPM] la cual es considerada de tipo estructural y la segunda es la pobreza monetaria [PM] más asociada con los ciclos económicos y por tanto de tipo, que como se dijo antes es indirecta y está fundamentada en el ingreso percápita familiar y en la línea de pobreza representada por el monto mínimo de ingreso que permite a un hogar disponer de recursos suficientes para atender las necesidades básicas (Martínez, 2015).

Así, la pobreza monetaria se determina a partir de los ingresos de los hogares los cuales provienen mayoritariamente del trabajo, tanto en América Latina como en Sincelejo, por lo cual se resalta la importancia que tiene sobre ella, tanto de la calidad del empleo como las oportunidades de trabajo del mercado y la magnitud de los ingresos laborales de los perceptores (CEPAL, 2019). Por tanto, de acuerdo con Banco Mundial -BM y el Banco Internacional de Reconstrucción y Fomento -BIRF (1990), la pobreza monetaria se encuentra íntimamente relacionada con el comportamiento de la actividad económica generadora de oportunidades de trabajo, así como también, según (Mincer, 1974), del nivel educativo de los trabajadores que a su vez incide sobre la magnitud de los ingresos laborales como proxy de la productividad laboral.

\section{Método}

Para calcular las probabilidades de que las personas sin condición de pobreza caigan en ella, se utilizan modelos econométricos de respuesta cualitativa, también denominados modelos probabilísticos. Aunque se cuenta con varios modelos alternativos para lograr los objetivos propuestos, en este trabajo se eligió el modelo de respuesta binaria tipo Logit en lugar del Probit ya que posibilita cálculos de manera más sencilla.

Por tanto, se realizarán dos regresiones que darán paso al mismo número de modelos estimados, la primera de ellas en la cual la variable dependiente corresponde a la pobreza cuando el perceptor principal, que casi siempre es el jefe del hogar, se queda sin trabajo y la otra que utiliza como variable dependiente a la pobreza proveniente de la falta de trabajo de los perceptores secundarios.

1 Para una ampliación se puede consultar además a López C., Hugo y Nuñez, Jairo (2007). Pobreza y desigualdad en Colombia. Diagnóstico y estrategias. Departamento Nacional de Planeación DNP. 


\subsection{El modelo Logit}

Tal como se indicó anteriormente, el Logit es un modelo de respuesta cualitativa que hace uso, para el cálculo de probabilidades, de la distribución logística en lugar de la distribución normal que maneja el modelo Probit. Para presentar el Logit, considérese inicialmente el modelo lineal.

$$
Y=C+\beta_{1} X_{1}+\beta_{2} X_{2}+\beta_{3} X_{3}+\cdots+\beta_{n} X_{n}+\varepsilon
$$

El cual puede presentarse de manera compacta como:

$$
\mathrm{Y}=\beta \mathrm{X}+\varepsilon
$$

Así, la probabilidad de que $\mathrm{Y}=1$ (es decir probabilidad de que el evento ocurra) y la probabilidad de que $\mathrm{Y}=0$ (es decir probabilidad de que el evento no ocurra), condicionado a los valores de las variables efecto o explicativas $\mathbf{X}$, de conformidad con (Green, 2008) y (Cabrer, Sancho \& Serrano, 2001), tiene las siguientes formas,

$$
\begin{aligned}
& \operatorname{Prob}(\mathrm{Y}=1)=\mathrm{P}=\mathrm{F}(\mathrm{X}, \beta) \\
& \operatorname{Prob}(\mathrm{Y}=0)=1-\mathrm{P}=1-\mathrm{F}(\mathrm{X}, \beta)
\end{aligned}
$$

Donde, F es la función de distribución acumulada logística, y por tanto,

$$
\begin{aligned}
& P=\operatorname{Prob}(Y=1)=F(X, \beta)=\left(\frac{1}{1+e^{-\beta X}}\right)=\left(\frac{e^{\beta X}}{1+e^{\beta X}}\right) \\
& 1-P=\operatorname{Prob}(Y=0)=1-F(X, \beta)=1-\left(\frac{e^{\beta X}}{1+e^{\beta X}}\right)=\left(\frac{1}{1+e^{\beta X}}\right)
\end{aligned}
$$

Así entonces, al realizar las operaciones aritméticas correspondientes se obtiene:

$$
\frac{P}{1-P}=e^{\beta x}
$$

De donde se concluye, al aplicar logaritmos naturales (Ln) de ambos lados de la ecuación [7] anterior, que

$$
L=\operatorname{Ln}\left(\frac{P}{1-P}\right)=\beta X
$$

Esta ecuación [8] representa el modelo a estimar, cuya variable explicada es el logaritmo de la razón de probabilidades. La razón de probabilidades es también denominada Odds ratio (Gujarati \& Porter, 2010). El modelo estimado y la probabilidad estimada vienen dados entonces por la ecuación [9] siguiente,

$$
\widehat{\mathrm{L}}=\operatorname{Ln}\left(\frac{\widehat{\mathrm{P}}}{1-\widehat{\mathrm{P}}}\right)=\operatorname{Ln}(\text { odds ratio) }=\hat{\beta} \mathrm{X}
$$

y

$$
\widehat{P}=\left(\frac{e^{\hat{\beta} X}}{1+e^{\beta X}}\right)
$$


En este caso, al estimar el Logit (L) se puede calcular la probabilidad de que $\mathrm{Y}=1$ dados unos valores específicos para las variables del vector $X$ aplicando la ecuación [10] anterior. El efecto marginal de un cambio de una unidad en la variable $X_{k}$ sobre $P$ viene dado por la siguiente expresión [11] (Gujarati \& Porter, 2010).

$$
\frac{\partial \mathrm{P}}{\partial \mathrm{X}}=\hat{\beta}_{\mathrm{k}}(\widehat{\mathrm{P}})(1-\widehat{\mathrm{P}})
$$

La estimación de los parámetros del modelo Logit se realiza mediante el método de Máximo Verosímil (MV) que garantiza, en muestras grandes como la usada en este trabajo, que los errores estándar estimados sean asintóticos. La significancia global del modelo se confirma rechazando la hipótesis nula de que todos los parámetros son iguales a cero de manera simultánea, utilizando la prueba de Razón de Verosimilitud (LR test o Likelihood Ratio) que se distribuye de manera asintótica como una Chi cuadrada. Para conocer la bondad de ajuste del modelo se utiliza tanto el Pseudo $\mathrm{R}^{2}$ (de McFadden) como la proporción de predicciones correctas (Gujarati \& Porter, 2010).

\subsection{Los datos}

Como se señaló antes, este trabajo utiliza la información proveniente de 545 encuestas tipo calidad de vida las cuales se aplicaron durante el mes de junio del año 2017, en el mismo número de hogares distribuidos en todo el territorio del municipio de Sincelejo. De estas, cuatro se descartaron por cuanto contenían errores. Con las 541 válidas se organizó la base de datos correspondiente la cual se instituye en el sustento estadístico de la presente investigación. Cada encuesta estaba constituida por 71 preguntas, algunas de ellas sobre características socioeconómicas y del jefe del hogar. Otras de las preguntas se referían a los ingresos, tanto de los provenientes de la actividad productiva como los no laborales de los hogares de Sincelejo encuestados, que permitieran totalizar sus ingresos y calcular el ingreso per cápita, de tal manera que se posibilitara su clasificación como pobre (monetariamente), en caso de ser inferior a los $\$ 232.555$ determinados como línea de pobreza por el DANE; y como no pobre en caso contrario. Esta misma información se utilizó para establecer la composición socioeconómica de la población según estratos propuestos por (CEPAL, 2019).

La forma en que se eligieron los 545 hogares sincelejanos encuestados fue la siguiente: 1. Hogares urbanos: Se utilizó un plano cartográfico de la ciudad de Sincelejo y se aplicó muestreo estratificado aleatorio simple, es decir, la cantidad de encuestas en igual proporción al conjunto de hogares de cada uno de los seis estratos socioeconómicos [oficiales en Colombia] de la capital sucreña, según los datos proporcionados por Electricaribe E. S. P. sobre la estratificación de los diferentes barrios de Sincelejo sobre los que se factura la energía consumida. 2. Hogares rurales: Sobre un listado de los corregimientos pertenecientes al municipio de Sincelejo, se eligieron aleatoriamente dos de ellos.

Por tanto, en esta investigación, el universo está constituido por el total de hogares del municipio de Sincelejo y la unidad muestral es cada hogar sincelejano. El error de muestreo de la encuesta aplicada se estimó en 4,2\% con un 95\% de confianza utilizando la proyección hecha por el (DANE, 2018) de 70.346 hogares, con corte junio de 2017.

Utilizando la base de datos conformada a partir de las encuestas aplicadas, se estimó un modelo Logit, en el cual, a la variable regresada (Pobre) se le asigna el valor uno (1) si el hogar se clasifica en pobreza monetaria, y cero (0) cuando ello no sucede. Por tratarse de información de corte transversal, 
para la estimación del modelo se utilizó la prueba de Huber/White disponible en el paquete Eviews 10.1, por lo cual los coeficientes estimados son robustos debido a la corrección de eventuales problemas de heteroscedasticidad.

\section{Resultados}

En esta sección se precisan las variables consideradas en cada uno de los dos modelos y se muestran los hallazgos estadísticos con un nivel de significancia del 5\%, que dan cuenta de la significancia estadística tanto del modelo considerado globalmente como de todos los coeficientes de manera individual lo que permite el cálculo de las probabilidades.

\subsection{Estimación y resultados del modelo empírico.}

Como se dijo antes, se estimaron dos modelos y por tanto la variables dependientes o explicadas serán: Para el modelo 1, Y=SINJE (pobreza si el perceptor principal se queda sin trabajo, categórica dicótoma), la cual toma el valor 1 si se trata de un hogar con en condición de pobreza por falta de ingresos del perceptor principal, y 0 en caso contrario. Para el Modelo 2, Y=SINOT (pobreza si los perceptores secundarios se quedan sin trabajo, categórica dicótoma), la cual toma el valor 1 si se trata de un hogar con en condición de pobreza por falta de ingresos de los perceptores secundarios, y 0 cuando ello no sucede. Las regresoras incorporadas corresponden a los cinco estratos socioeconómicos vulnerables que son: bajo no pobre, medio bajo, medio intermedio, medio alto y alto, todas ellas categóricas dicótomas que toman el valor de 1 si el hogar pertenece al estrato correspondiente y 0 en caso contrario. Así las cosas, se tiene que:

- Variables dependientes: SINJE (Modelo 1) y SINOT (Modelo 2)

- Variables independientes: BAJO, MEBAJO, MEINTER, MEALTO Y ALTO.

Por tanto, los modelos que se estimarán vienen dados por la ecuación [12]:

$$
\mathrm{L}=\operatorname{Ln}\left(\frac{\mathrm{P}}{1-\mathrm{P}}\right)=\beta_{0}+\beta_{0} \mathrm{MEBAJO}+\beta_{0} \text { MEINTER }+\beta_{0} \text { MEALTO }+\beta_{0} \text { ALTO }
$$

En donde la categoría base utilizada para estos modelos es la del estrato bajo (BAJO). Los resultados obtenidos al realizar las dos regresiones para estimar los modelos 1 y 2, se indican en la Tabla 2. En este punto es importante anotar que para estimar el modelo 2 no se tuvieron en cuenta las variables MEALTO y ALTO debido a que ninguno de los individuos de los estratos Medio Alto y Alto registra pobreza por falta de ingresos de los perceptores secundarios y por tanto el número de observaciones se reduce a 506 debido a la exclusión de los hogares que pertenecen a estos estratos. 
TABLA 2. RESULtAdOS DE REGRESIÓN MODELOS 1 Y 2

\begin{tabular}{|c|c|c|c|}
\hline \multirow[t]{2}{*}{ Descripción/Nombre } & Variables & Coeficientes Modelo 1 & $\begin{array}{l}\text { Coeficientes Mod- } \\
\text { elo } 2\end{array}$ \\
\hline & Intercepto & $2,194511(*)$ & $\left.1,2653811^{*}\right)$ \\
\hline Estrato Medio Bajo & Mebajo & $-2,509592(*)$ & $-2,632258(*)$ \\
\hline Estrato Medio Intermedio & Meinter & $-3,027420(*)$ & $-4,731117\left(^{*}\right)$ \\
\hline Estrato Medio Alto & Mealto & $-4,140421(*)$ & - \\
\hline Estrato Alto & Alto & $-5,084883(*)$ & - \\
\hline \multicolumn{2}{|c|}{ R2 Mcfadden } & 0,314134 & 0,223645 \\
\hline \multicolumn{2}{|c|}{ Estadístico Lr } & 189,5648 & 145,3674 \\
\hline \multicolumn{2}{|c|}{ Prob (Estadístico Lr) } & 0,000000 & 0,000000 \\
\hline \multicolumn{2}{|c|}{ Tamaño De La Muestra } & 541 & 506 \\
\hline \multicolumn{2}{|c|}{ Porcentaje De Predicciones Correctas } & $85,03 \%$ & $79,45 \%$ \\
\hline$(*)=\operatorname{Sig} 1 \%-(* *)=\operatorname{Sig} 5 \%-(* * *)=$ & & & \\
\hline
\end{tabular}

Así mismo, los modelos ajustados resultan adecuados, por cuanto:

$\gg$ Las variables predictoras de ambos modelos, consideradas individualmente, así como sus correspondientes interceptos, resultan ser estadísticamente significativas con una confianza de casi el $100 \%$ (Prob $=0,0000$ ) en cada uno de los dos modelos.

$\gg$ El R2 (de McFadden) para el modelo 1 resulta superior a 0,31, en tanto que para el modelo 2 en mayor que 0,22 , revelando el grado de la bondad del ajuste, pero teniendo en cuenta que la medida de $\mathrm{R}^{2}$ no es "particularmente significativa para los modelos con regresada binaria" como en este caso (Gujarati \& Porter, 2010).

$\gg$ Ambos modelos, en su conjunto, resultan estadísticamente significativos debido a los resultados del estadístico de Razón de Verosimilitud (LR) que tiene una probabilidad asociada [p-valor] menor de 0,05, por tanto, todos los coeficientes de pendiente de los dos modelos no pueden ser simultáneamente iguales a cero, con lo cual se concluye la significancia estadística de los modelos globalmente considerados con una confianza de casi el 100\% (Prob = 0,0000).

$\gg$ El valor de Cuenta $\mathrm{R}^{2}$ para el modelo 1 es de $85,03 \%$ y de $79,45 \%$ para el modelo 2 , lo que indica el alto porcentaje de predicciones correctas con relación al total de observaciones. De esta manera se ratifica la bondad del ajuste del modelo utilizado. 


\section{Discusión}

Con base en los resultados obtenidos se procedió a calcular las probabilidades en cada uno de los dos casos [perceptor principal sin ingresos laborales y perceptores secundarios sin ingresos laborales], utilizando para ello la ecuación [10], quedando como se indica en la figura 4 de la siguiente manera:

Figura 4. Riesgo de caer en situación de pobreza en Sincelejo

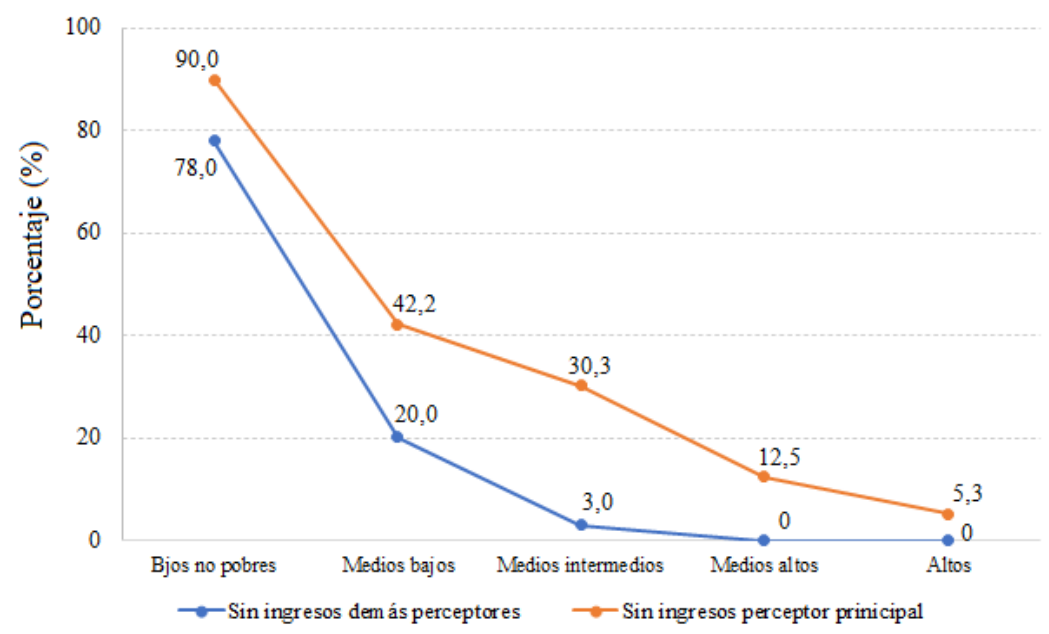

Fuente: Elaboración propia a partir de los resultados de Eviews 10.1 (2020)

La evidencia empírica ratifica la importancia de los ingresos laborales de los perceptores principales del municipio de Sincelejo, en el sentido de que su ausencia provoca mayores probabilidades de que los individuos caigan en situación de pobreza, que aquellos en los que faltan de ingresos los de los perceptores secundarios. Igualmente se establece que, para ambos casos, bien sea de ausencia de ingresos laborales del perceptor principal o de los perceptores secundarios, las probabilidades de caer en pobreza disminuyen en la medida en que los estratos sean más altos y por supuesto entonces, los grados de vulnerabilidad de los individuos tienen relación inversa con los estratos socioeconómicos de tal manera que el estrato bajo no pobre resulta ser el de mayor vulnerabilidad. Por otra parte, estos resultados son compatibles con los obtenidos para América Latina por parte de (CEPAL, 2019), en el sentido de que tienen el mismo comportamiento, añadiendo que los valores de las probabilidades de caer en situación de pobreza en Sincelejo superan en todos los casos y estratos a los de la región.

De cumplirse las proyecciones presentadas en el grafico siguientes, el estado colombiano no ofrecería mucho apoyo, pues, el marco jurídico de Colombia es relativamente nuevo en la defensión y protección del derecho al trabajo, por lo cual se creería que la población sincelejana podría quedar en estado de vulnerabilidad a causa de la falta de ingreso para satisfacer sus necesidades básicas. No obstante, de la normativa jurídica que ayudaría a mitigar el impacto de esta problemática se concentrarían en el apoyo al cesante (ley 1636 de 2016) por medio del auxilio que ofrecen las cajas de compensaciones y el ingreso de manera oportuna a la oficina de empleo para conocer las vacantes en el mercado laboral (ley 789 de 2002) 


\section{Conclusiones}

La situación del municipio de Sincelejo en materia de pobreza monetaria se puede calificar de grave considerando el alto valor de su índice correspondiente, que supera 1,5 veces al de Colombia por cuanto se ubica en el $44 \%$ en contraste con el $28,9 \%$ nacional, así como también se encuentra muy por encima de los valores de un país como Uruguay que solo registra el 2,7\% de pobreza en el año 2017.

También resulta preocupante la alta participación del estrato bajo no pobre en la población total de Sincelejo, del orden del 37,7\% que también supera el promedio nacional y en 1,5 veces el de América Latina; por cuanto se trata de la población con más alta vulnerabilidad de conformidad con los resultados obtenidos en el presente trabajo con una probabilidad del $90 \%$ de caer en pobreza por la falta de ingresos del perceptor principal y del $78 \%$ ante la ausencia de ingresos laborales de los perceptores secundarios.

Esta situación se viene presentando a nivel mundial y de manera masiva en el año 2020, particularmente en Colombia y todas sus regiones desde el mes de mayo, por cuenta de la destrucción en mayo de 2020 con relación al promedio del trimestre dic19-feb20, según (DANE, 2020), de 4 millones de puestos de trabajo ocasionada por la fuerte contracción del PIB, estimada para 2020 por (FEDESARROLLO, 2020) entre el 2,7 y el 7,9\%; provocada por la pandemia generada por el Covid 19. Ello tendrá como consecuencia que en el presente año se profundizará la grave situación de pobreza de Sincelejo elevando sustancialmente tanto el número de pobres como los índices de pobreza monetaria que pueden sobrepasar el $70 \%$, por lo cual se estima que los avances obtenidos en materia de pobreza en los últimos años se esfumarán, ya que según el Fondo monetario Internacional - (IMF, 2020), aunque el crecimiento económico de las economías avanzadas sufrirán mayores caídas que las emergentes y en desarrollo, estas serán del orden del 3\%, así como también señala que "el efecto adverso sobre las familias de ingreso bajo será especialmente agudo, lo que compromete el significativo progreso conseguido en el mundo en la reducción de la pobreza extrema desde los años noventa." (p. 8).

Sin embargo, (Boltvinick \& Damián, 2020) tienen predicciones más pesimistas sobre la magnitud del colapso que provocará el Covid 19, pues considera que tanto la caída del PIB como el incremento de la pobreza se han calculado con base en "la elasticidad-pobreza de la caída del PIB en recesiones anteriores", las cuales consideran insuficientes; por lo cual creen que "El daño humano de esta pobreza es enorme, probablemente mayor al daño directo de la pandemia" (p.9).

Por su parte (OXFAM, 2020), con relación al incremento en la pobreza, afirma que el mundo contará con 500 millones de pobres adicionales y en las 13 principales ciudades de Colombia, según estimaciones de (Sánchez \& Chaparro, 2020), la incidencia de la pobreza se incrementará pasando del 18\% al 32\%, en tanto que la de pobreza extrema pasará de 4,5\% a 16,7\%. Es importante resaltar que, para estos autores, la población vulnerable es aquella que proviene de hogares no pobres en los que el ingreso percápita está por debajo del ingreso mediano, que para el trimestre IV de 2019 se ubicó en $\$ 626.000$ en las 13 ciudades.

Los antecedentes históricos y la actualidad económica de Colombia y de otros países del mundo permiten evidenciar que las leyes no son suficientes para extinguir o reducir la pobreza monetaria a una mínima expresión. Se requieren de unas virtudes y valores en una sociedad, que se antepongan a la codicia de un sector de la población, a la avaricia de otro sector y a la holgazanería de quienes teniendo las facultades físicas y mentales para trabajar se niegan a hacerlo. Se requiere romper con esos vicios que (Cunningham y Rogers, 2010) denominan trinidad maligna de la codicia, corrupción e injusticia. Para estos autores, cualquier país puede prosperar si sus habitantes se esfuerzan, ahorran, mantiene tipos de interés razonable y viven bajo el sistema de la ley y la responsabilidad. De lo contrario, el resultado será esclavitud, injusticia y pobreza (Beliles, 2017). 
Por todo lo anterior, se hace necesario la aplicación de políticas públicas efectivas tendientes, no solo las enfocadas en la población en condición de pobreza, sino las encaminadas a fortalecer a la población más vulnerable de tal manera que puedan afrontar de una mejor manera los eventos económicos adversos como los inducidos por la pandemia generada por la COVID 19 y de esa manera evitar su caída a la pobreza, de tal manera que ante tales fenómenos se pueda moderar o impedir el preocupante aumento de la población pobre y el deterioro de los índices de pobreza.

\section{Referencias Bibliográficas}

Acemoglu, D. y Robinson, J. (2012). Por qué fracasan las naciones. Barcelona: Planeta.

Anaya Narváez, A., Buelvas, J. \& Valencia, L. (2015). “Modelo Probit para la medición de la pobreza en Montería, Colombia". Revista Opción. Año 31, № 78, pp 42-64.

Banco Mundial y Banco Internacional de Reconstrucción y Fomento. (1990). Resumen del Informe sobre desarrollo mundial 1990. En http://documentos.bancomundial.org/curated/es/ docsearch?query=Resumen\%20del\%20Informe\%20sobre\%20desarrollo\%20mundial\%201990

Banco Mundial. (1990). World Develoment Report: poverty. Washington DC: WB.

Barba, C. (2009). Los estudios sobre la pobreza en América Latina. Revista Mexicana de. Sociología, Vol.71, número especial, pp. 9-49.

Beliles, M. (2017). Transformando las naciones. Santa Cruz, Bolivia. Red de transformación global latinoamericana.

Boltvinik, J. \& Damián, A. (2020). El Covid-19 está aumentando mucho la pobreza y la desigualdad. Economía Unam, 18, (51), pp 374-385.

Busso, G. (2005). Pobreza, exclusión y vulnerabilidad social. Usos, limitaciones y potencialidades para el diseño de políticas de desarrollo y de población. En: https://www.academia.edu/3887686/POBREZA_ EXCLUSI\%C3\%93N_Y_VULNERABILIDAD_SOCIAL_Usos_limitaciones_y_potencialidades_para_el_ dise\%C3\%B10_de_pol\%C3\%ADticas_de_desarrollo_y_de_poblaci\%C3\%B3n

Cabrer Borrás, B., Sancho Pérez, A., \& Serrano, G. (2001). Microeconometría y decisión. Madrid: Pirámide.

Casas, J. \& Barichello, R. (2015). Hacia una noción sobre la pobreza. Apuntes del CENES. 34 (59), pp. 39-62

Castelao, O., Massot, J. \& Eriz, M. (2014). Riesgo social: medición de la vulnerabilidad en grupos focalizados. Cuadernos Del CIMBAGE, (16), 27-51.

CEPAL (2019). Panorama Social de América Latina. En https://repositorio.cepal.org/bitstream/ handle/11362/44969/5/S1901133_es.pdf (fecha de consulta 3006 2020).

Colson, C. \& Pearcey, N. (2000). Y ahora... ¿cómo viviremos? México: Unilit.

Cope, L (2010). El modelo de transformación social del Antiguo Testamento. Texas, USA: JUCUM.

Cunningham, L. y Janice, R. (2010). El libro que transforma naciones. Tyler, EE. UU: JUCUM. ISBN: 9781576584286

DANE (2018). Series de población. En: http://www.dane.gov.co/index.php/estadisticas-por-tema/demografia$\mathrm{y}$-poblacion/series-de-poblacion

DANE (2020). Pobreza y Desigualdad. En: https://www.dane.gov.co/index.php/estadisticas-por-tema/ pobreza-y-condiciones-de-vida/pobreza-y-desigualdad

Durán Gil, C. A. (2017). Análisis espacial de las condiciones de vulnerabilidad social, económica, física y ambiental en el territorio colombiano. Perspectiva Geográfica, 22(1). doi: 10.19053/01233769.5956. 
FEDESARROLLO (2020). Comunicado de prensa FEDESARROLLO de abril 21 de 2020. https://www.fedesarrollo. org.co/

Green, W. (2008). Análisis Econométrico. Madrid: Prentice Hall.

Gujarati, D., \& Porter, D. (2010). Econometría. México: Mc Graw Hill.

IMF (2020). “World Economic Outlook Update, June 2020”: https://www.imf.org/ en/Publications/WEO / Issues/2020/06/24

Jaimez, R. (2016). La investigación de la pobreza, la ciencia social emancipatoria y el pensamiento crítico. Acta Sociológica, N³0, pp. 29-48. https://doi.org/10.1016/j.acs0.2017.01.002

Klisberg, B. (2011). Mitos sobre la pobreza. En http://repositoriouba.sisbi.uba.ar/gsdl/collect/encruci/index/ assoc/HWA_128.dir/128.PDF

Mangalwadi, V. (2009). Verdad y transformación. Seattle, USA: JUCUM.

Marduk, S., González, M. \& Malavé, J. (2010). Percepciones acerca de los pobres y la pobreza: Una revisión. Revista Latinoamericana de Psicología, 42 (3), pp. 413-425

Martínez, B. (2015). Planteamientos sobre la pobreza: una aproximación conceptual. Apuntes Cenes. 34 (60), pp. $15-40$

Mayorga, A. (2018). Debates contemporáneos sobre la pobreza: entre causas, representaciones y programas sociales. Trabajo Social 20 (1): 163-193. Bogotá: Departamento de Trabajo Social, Facultad de Ciencias Humanas, Universidad Nacional de Colombia.

Merchan, C. 2011). El ABC de la Ley de formalización y generación de empleo. Ministerio de la Protección Social, Dirección General de Salud Pública.

Miller, D. (2001). Discipulando Naciones: el poder de la verdad para transformar culturas. Managua, Nicaragua: EMCOR.

Miller, D. (2011). Vida, trabajo y vocación. Tyler, USA: JUCUM.

Mincer, J. (1974). Schooling, experience and earnings. NBER Columbia University Press, 1974, 71-94.

Miranda Delgado, R. (2020). Pobreza, desigualdad y vulnerabilidad en América Latina. Americanía. Revista de Estudios Latinoamericanos. Nueva Época (Sevilla), n. 11, p. 56-90.

Mora, M. \& Pérez, J. (2006). De la vulnerabilidad social al riesgo de empobrecimiento de los sectores medios: un giro conceptual y metodológico. Estudios Sociológicos, XXIV, (70), 99-138.

Moser, C. (1999). Marco conceptual sobre activos, vulnerabilidad y estructura de oportunidades (Primera). Montevideo: CEPAL.

Naciones Unidas (2004): Informe sobre la situación social del mundo 2003. Vulnerabilidad social: Fuentes y desafíos. Nueva York: United Nations Publications.

Nicholson, W., \& Snyder, C. (2015). Teoría Microeconómica: Principios básicos y ampliaciones. México, D.F.: Cengage Learning.

Núñez, J., \& Espinosa, S. (2005). No siempre pobres, no siempre ricos: Vulnerabilidad en Colombia (Documento CEDE 2005-15). Bogotá.

Oxfam International, "The hunger virus: How covid-19 is fuelling hunger in a hungry world", 9 de julio de 2020, 21 pp: www.oxfamilibrary.openrepo sitory.com

Perloff, J. M. (2012). Microeconomics, Theory and Applications with Calculus. Harlow, England: Pearson. República de Colombia (1991). Constitución Política. Bogotá: Leyer. 
República de Colombia. (2010). Ley 1429. Recuperado de https://www.sic.gov.co/sites//default/files/normatividad/ Ley_pdf1429_2010.pdf

República de Colombia. Congreso Nacional de la República. Ley 1636. (18 de junio de 2013). Por medio de la cual se crea el mecanismo de protección al cesante en Colombia

República de Colombia. Congreso Nacional de la República. Ley 1780 (2 de mayo de 2016). Por medio de la cual se promueve el empleo y el emprendimiento juvenil, se generan medidas para superar: barreras de acceso al mercado de trabajo y se dictan otras disposiciones.

República de Colombia. Congreso Nacional de la República. Ley 789. (27 de Diciembre de 2002). Por la cual se dictan normas para apoyar el empleo y ampliar la protección social y se modifican algunos artículos del Código Sustantivo del Trabajo. Diario Oficial No. 45.046. Bogotá, D.C.: Imprenta Nacional de Colombia

Sachs, J. (2008). El fin de la pobreza: cómo conseguirlo en nuestro tiempo. Barcelona: Debate.

Sánchez Torres, R \& Chaparro Hernández, S. (2020). Un piso de protección social para preservar la vida: Informalidad, pobreza y vulnerabilidad En tiempos de covid-19. Centro de Investigaciones para el Desarrollo -CID. FCE - CID № 35

Sen, A. (2000). Desarrollo y Libertad. Barcelona, España: Planeta.

Wood, N. J., Burton, C. G., \& Cutter, S. L. (2010). Community variations in social vulnerability to Cascadiarelated tsunamis in the U.S. Pacific Northwest. Natural Hazards, 52(2), 369-389. https://doi.org/10.1007/ s11069-009-9376-1 